\title{
Best Practice Recommendations for Dysphagia Management in Stroke Patients: A Consensus from a Portuguese Expert Panel
}

\author{
Isabel de Jesus Oliveira ${ }^{a, b} \quad$ Germano Rodrigues Couto ${ }^{c, d}$ Rosa Vilares Santos ${ }^{d, e}$ \\ Ana Maria Campolargo ${ }^{f}$ Cláudia Lima ${ }^{f}$ Pedro Lopes Ferreirab, $g$

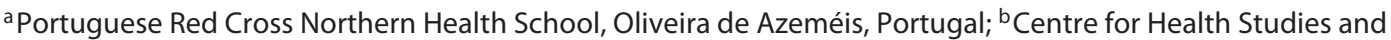 \\ Research of the University of Coimbra, Coimbra, Portugal; ' ${ }^{C}$ Health School of the University of Fernando Pessoa,

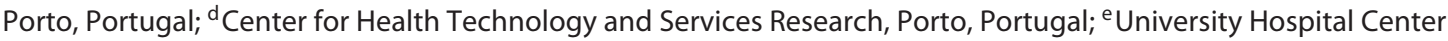 \\ of São João, Porto, Portugal; ${ }^{f}$ Hospital Center Vila Nova de Gaia/Espinho, Vila Nova de Gaia, Portugal; ${ }^{9}$ Faculty of \\ Economics of the University of Coimbra, Coimbra, Portugal
}

\section{Keywords}

Dysphagia · Stroke - Disease management - Therapeutics · Recommendations

\begin{abstract}
Dysphagia is frequent after stroke, and it increases the risk of respiratory infection, dehydration and malnutrition, resulting in worse outcomes. Different clinical guidelines present recommendations for the assessment and management of dysphagia in stroke patients in a scattered way. These best practice recommendations address seven clinical questions on the assessment and management of dysphagia in stroke patients, gathering the best-updated evidence. A systematic literature review using the PICO strategy was performed. The recommendations draft was then appraised by a multidisciplinary panel of experts (nutritionists, physiatrists, speechlanguage pathologists and rehabilitation nurses) in a total of 3 Delphi rounds. A minimum of $80 \%$ consensus was established, and the final version offers a total of 21 recommendations for use in clinical practice for stroke patients. These clinical recommendations are an overview of the most recent evidence combined with experts' consensus and translated into clinically relevant statements. In implementing recom-
\end{abstract}

mendations at the local level, health professionals should identify facilitators and barriers to evidence-based practice within their contexts and determine the best strategies to address local needs. Where the change is needed, initial and continuing training on all recommendations is essential and relevant.

(c) 2022 The Author(s). Published by S. Karger AG, Basel on behalf of NOVA National School of Public Health

\section{Boas práticas no tratamento da disfagia em doentes com AVC: consenso de peritos portugueses}

\section{Palavras Chave}

Disfagia · Acidente vascular cerebral · Gestão da doença • Tratamento · Recomendações

\section{Resumo}

A disfagia é frequente após o acidente vascular cerebral e aumenta o risco de infecção respiratória, desidratação e desnutrição, resultando em piores resultados em saúde. Diferentes diretrizes clínicas apresentam recomendações para a avaliação e tratamento da disfagia em doentes com acidente vascular cerebral de forma dispersa. Estas reco- (c) 2022 The Author(s). Published by S. Karger AG, Basel on behalf of NOVA National School of Public Health

This is an Open Access article licensed under the Creative Commons Attribution-NonCommercial-4.0 International License (CC BY-NC) (http://www.karger.com/Services/OpenAccessLicense), applicable to the online version of the article only. Usage and distribution for commercial purposes requires written permission.
Correspondence to:

Pedro Lopes Ferreira, pedrof@fe.uc.pt 
mendações de melhores práticas abordam sete questões clínicas sobre avaliação e tratamento da disfagia em doentes com acidente vascular cerebral, reunindo a evidência mais atualizada. Para responder a estas questões foi realizada uma revisão sistemática da literatura usando a estratégia PICO. O projeto de recomendações foi então submetido à apreciação de um painel multidisciplinar de peritos (nutricionistas, fisiatras, terapeutas da fala e enfermeiros especialistas em enfermagem de reabilitação) num total de três rondas Delphi. Foi estabelecido um consenso mínimo de $80 \%$ e a versão final apresenta um total de 21 recomendações para uso na prática clínica para doentes com acidente vascular cerebral. Estas recomendações clínicas são uma visão geral da evidência mais recente combinada com o consenso de peritos e traduzidas em declarações clinicamente relevantes. Ao implementar as recomendações ao nível local, os profissionais de saúde devem identificar facilitadores e barreiras para a prática baseada na evidências em seus próprios contextos e determinar quais as melhores estratégias para responder às necessidades locais. Onde a mudança é necessária, a formação inicial e contínua em todas as recomendações é essencial e relevante.

๑ 2022 The Author(s). Published by S. Karger AG, Basel on behalf of NOVA National School of Public Health

\section{Introduction}

Stroke represents the second cause of death in the world [1] and the second cause of years of healthy life lost due to disability [2]. One of its more frequent complications is dysphagia, which can reach $80 \%$ in the acute phase [3]. There is an unequivocal relation between dysphagia and respiratory complications [4] as well as increased risk of dehydration [5] and malnutrition [6]. These complications result in an increased length of stay, institutionalization, mortality and poorer functional outcomes. In addition to the impact on the patient, it also has a great impact on health costs, with an increase of $40.36 \%$ [7].

Several clinical guidelines list in a dispersed way recommendations for assessment and management of dysphagia after stroke, making it difficult to translate evidence to clinical practice. Therefore, it is essential to develop specific clinical guidelines that support good practice for dysphagia management in stroke patients, from onset to rehabilitation. The framing, implementation and utilization of clinical guidelines, grounded on the best available evidence and adjusted to clinical con- texts, guarantee the quality and excellence of care provided to stroke patients.

The objective of this work is to provide health professionals with a set of clinical recommendations for the therapeutic approach to stroke patients with dysphagia to streamline the evidence-based practice.

\section{Methods}

The World Health Organization [8] guidelines for the development of clinical practice recommendations were used. A preliminary literature search allowed to narrow and define accurately the areas which researchers aimed to cover with the recommendations. Experts were also consulted to clarify and focus research on the scope of practice. Three investigators defined the areas that the set of recommendations should cover, as well as the target population (stroke patients) and clinical condition (dysphagia), from acute to rehabilitation stage, covering diagnostic and therapeutic evidence-based interventions that decrease morbidity and mortality or improve outcomes. From there, seven clinical questions were formulated in PICO format.

Then, the methodology for a systematic literature review was defined, which included the search for clinical guidelines, systematic literature reviews/meta-analysis and experimental or quasiexperimental studies and observational studies, published between 2008 and 2019 and in Portuguese, English and Spanish, in the following databases: Cochrane Library; CINAHL with Full Text; PubMed complemented by manual search on pages of scientific/ professional societies in related areas and entities responsible for issuing clinical guidelines. Database search was conducted during 2019. Due to the complexity of the literature search, an example of the search strategy is shown in Table 1 and the full search strategy is available in the supplementary material (for all online suppl. material, see www.karger.com/doi/10.1159/000520505).

The clinical guidelines found were analyzed to identify relevant content and selected accordingly. Then, systematic reviews and meta-analyses were searched. The search for experimental/quasiexperimental or observational studies was dependent on the recovered reviews and the evaluation of its methodological quality. Depending on the publication date, the search was repeated for the subsequent period, in an attempt to identify studies published for further analysis.

Regarding the assessment of methodological quality, it was carried out by two independent researchers. AGREE II was used for the assessment of clinical guidelines [9], and those that obtained an average score in all domains $\geq 70 \%$ were considered for inclusion. The assessment of the methodological quality of systematic reviews was guided by AMSTAR 2 [10] and moderate to highquality reviews were included. For primary research, the tools provided by the Critical Appraisals Skills Program were used [11].

For the preparation of the first draft of recommendations, clinical guidelines and/or systematic reviews of high methodological quality were used directly, as long as they were updated. The existence of high quality and/or relevant experimental or quasi-experimental studies published after the publication of clinical guidelines determined their analysis and inclusion for discussion by experts. At the end of this phase, the level of evidence (LOE) was 
Table 1. Example of literature search strategy

\begin{tabular}{ll}
\hline Clinical question & $\begin{array}{l}\text { Does modifying food and liquid consistency, compared to standard feeding, decrease mortality and } \\
\text { morbidity, or improve health outcomes in stroke patients? }\end{array}$ \\
\hline Population & Stroke patients with dysphagia \\
\hline Intervention & Modification of food consistencies and liquid viscosity \\
\hline Comparison & Standard or nonmodified feeding \\
\hline Outcome & Efficacy and safety of swallowing and quality of life \\
\hline Search terms & $\begin{array}{l}\text { Stroke; cerebrovascular accident; apoplexy; dysphagia; swallowing disorders; liquid; food; texture; con- } \\
\text { sistency; thick*; viscosity }\end{array}$ \\
\hline First search & $\begin{array}{l}\text { Three guidelines: Burgos et al. [21], 2018; Stroke Foundation [20], 2019; Wirth et al. [27], 2013 } \\
\text { Four systematic reviews: Beck et al. [93], 2018; Newman et al. [94], 2016; Steele et al. [100], 2015; Swan et } \\
\text { al. [101], 2015 }\end{array}$ \\
\hline $\begin{array}{l}\text { Second search for updated } \\
\text { evidence }\end{array}$ & $\begin{array}{l}\text { Five observational studies: Bolivar-Prados et al. [102], 2019; Crary et al. [95], 2016; McCurtin et al. [98], } \\
\text { 2018; Miles et al. [96], 2018; Vilardell et al. [103], 2016 }\end{array}$ \\
\hline
\end{tabular}

rated according to Halperin et al. [12]. LOE level A is determined by high-quality evidence from more than one randomized controlled trial (RCT), meta-analyses of high-quality RCTs or one or more RCTs corroborated by high-quality registry studies; LOE level B-R is from moderate-quality evidence from one or more RCTs or meta-analyses of moderate quality RCTs; LOE level B-NR emerges from moderate-quality evidence from one or more welldesigned, well-executed nonrandomized studies, observational studies or registry studies or meta-analyses of such studies; LOE level C-LD by randomized or nonrandomized observational or registry studies with limitations of design or execution or metaanalyses of such studies or physiological or mechanistic studies in human subjects, and LOE level C-EO is supported on a consensus of expert opinion based on clinical experience [12]. After this process, a first draft was elaborated with a set of 23 recommendations for submission to a panel of experts in a Delphi-type technique by e-mail. This technique was intended to develop an expert-based judgment, based on the assumption that a group of experts and a variety of perspectives will produce a more valid result than just an individual. Each expert was asked to indicate the level of agreement for each recommendation (agree, partially agree or disagree), and for the responses "partially agree" and "disagree" they were asked to justify their answer on clinical/scientific grounds. The consensus was defined for levels of agreement above $80 \%$. Regarding the recommendation level, which concerns the magnitude of the benefit over the risk, an account was taken of the LOE existing for each recommendation and the degree of agreement and justification presented by each of the experts. The rating was performed according to Halperin et al. [12]: class of recommendation (COR) I (strong), when the treatment/procedure/intervention is useful and effective and should be provided to most patients under most circumstances; COR IIa (moderate), lower benefit over risk than COR I; COR IIb (weak), where the benefit is marginally higher than the risk; COR III, when the treatment/procedure/intervention is not recommended [12]. COR and LOE were assessed independently. Halperin et al. [12] argue that any COR can be paired with any LOE. A LOE of limited data does not imply that the rec- ommendation is weak. On one hand, experts may consider that, based on their clinical experience, the clinical benefit of an intervention may be evident. On the other hand, a specific intervention may not be suitable to be tested in a randomized controlled trial, making evidence weak, conflicting or absent in which cases authors advocate that guidance is foremost needed [12]. Therefore, the classification of the recommendation was based on the available evidence and its clinical relevance. It was also defined that only levels of agreement above $90 \%$ would allow classification up to class I (strong) and that levels of agreement above $80 \%$ but $\leq 90 \%$ would only have, at most, class IIa classification (moderate).

Recognizing that the Delphi technique is not a research method aligned with any specific methodology, the difficulty in defining quality criteria for its development that are widely accepted is highlighted [13]. In this sense, and to reduce the risk of habitus mentalis, experts from different professional areas, from different contexts of practice, both clinical and academic, were invited to participate in the purposive sampling technique. Diversity in a group of experts favors a more innovative discussion, thus becoming as relevant to the final result as the skills and expertise of the participants, enhancing the robustness and validity of the findings. Regarding the number of experts to invite, the available evidence is not consensual, with the number of experts varying from 3 to 713 , with an average of 14 [13]. For the Delphi panel of this study, 16 more easily accessible experts who agreed to participate were invited: 4 specialist nurses in rehabilitation nursing, 1 neurologist, 5 physiatrists, 2 nutritionists and 4 speech-language pathologists (SLPs). All experts had more than 10 years of effective clinical and/ or academic experience in the field of stroke. All experts were previously contacted to clarify the type and terms of their participation. Regarding ethical considerations related to the Delphi panel, the experts were also asked to declare any type of conflict of interest. The experts had no declared affiliation that could be perceived as posing a potential conflict of interest in the participation in this Delphi panel. The anonymity of the participants was also ensured. No patients were involved in the experts' panel. 
Table 2. Profile of experts invited to the Delphi panel

\begin{tabular}{|c|c|}
\hline Expert & Professional profile \\
\hline Rehabilitation nurse 1 & Academic/researcher \\
\hline Rehabilitation nurse 2 & Clinical practice - acute care \\
\hline Rehabilitation nurse 3 & Clinical practice - rehabilitation care \\
\hline Rehabilitation nurse 4 & Clinical practice - rehabilitation care \\
\hline Physiatrist 1 & Clinical practice - acute care \\
\hline Physiatrist 2 & Clinical practice - rehabilitation care \\
\hline Physiatrist 3 & Clinical practice - rehabilitation care ${ }^{1}$ \\
\hline Nutritionist 1 & Clinical practice - acute care/academic/researcher \\
\hline Nutritionist 2 & Clinical practice - rehabilitation care \\
\hline Speech-language pathologist 1 & Clinical practice - acute care/academic/researcher \\
\hline Speech-language pathologist 2 & Clinical practice - acute care \\
\hline Speech-language pathologist 3 & Clinical practice - rehabilitation care \\
\hline Speech-language pathologist 4 & Clinical practice - rehabilitation care ${ }^{1}$ \\
\hline
\end{tabular}

${ }^{1}$ Did not participate in the second and third rounds.

\section{Results}

The systematic review of the literature and manual research resulted in a first draft of 23 recommendations. None of the guidelines already published answered all the clinical questions initially formulated. The draft with the 23 recommendations was then submitted to the expert's appraisal. Of the 16 invited experts, 13 responded: 4 rehabilitation nurses, 3 physiatrists, 2 nutritionists and 4 SLPs (Table 2).

In the first round, a consensus was obtained for 14 recommendations and of these, 9 reached a consensus of over $90 \%$. Recommendations that reached a consensus level (in the sum of total and partial consensus) above $80 \%$, but below $90 \%$, went on to the next round with the incorporation of the experts' suggestions. Three recommendations reached consensus levels (in the sum of total and partial consensus) below $80 \%$ and were therefore rejected. For the second round, 7 recommendations were sent to the experts and an additional one emerged from the expert's opinion. To clarify some of the issues pointed at by the experts in the previous round, additional information was sent explaining the rationale for decisionmaking. In this round, all recommendations reached a level of consensus higher than $80 \%$.

The final version of the recommendations was sent to the experts, with an indication of the level of consensus obtained for each of them and the level of recommendation, for final validation. After the appraisal, a final version with 21 recommendations was obtained (Table 3).

\section{Clinical Question}

When should the ability to swallow be screened and assessed in stroke patients?

\subsection{Recommendation Statement(s)}

It is recommended in all services and to all patients admitted with a diagnosis of stroke (confirmed or not), in the acute phase, to implement a dysphagia screening protocol. It is recommended that screening be performed as early as possible, before any liquid, food or medication ingestion.

\section{COR I}

\section{LOE C-LD}

It is recommended that all patients with positive screening for dysphagia, or present other risk factors, undergo clinical evaluation by properly trained professionals. It is reasonable, whenever possible, to give preference to instrumental assessment, considering the availability of technical and human resources.

\section{COR IIa}

\section{LOE C-EO}

It is recommended that screening for dysphagia should be carried out by a properly trained nurse, doctor or SLP.

\section{COR I \\ LOE C-EO}

\subsection{Summary of Evidence}

For dysphagia assessment, more specifically for the identification of aspiration (passage of material to the larynx - food or liquids - below the level of the vocal folds), instrumental evaluation (videofluoroscopy swallowing study) has been considered the gold standard [14]. Ide- 
Table 3. Summary of recommendations

\begin{tabular}{|c|c|c|c|}
\hline & & $\begin{array}{l}\text { Class of } \\
\text { recommendation }\end{array}$ & $\begin{array}{l}\text { Level of } \\
\text { evidence }\end{array}$ \\
\hline Recommendation 1 & $\begin{array}{l}\text { It is recommended that in all services and to all patients admitted with a } \\
\text { diagnosis of stroke (confirmed or not), in the acute phase, a dysphagia } \\
\text { screening protocol should be instituted. Screening should be performed as } \\
\text { early as possible, before any liquid, food or medication ingestion }\end{array}$ & I & C-LD \\
\hline Recommendation 3 & $\begin{array}{l}\text { It is recommended that all patients with positive screening for dysphagia, } \\
\text { or present other risk factors, undergo clinical evaluation by properly } \\
\text { trained professionals. It is reasonable, whenever possible, to give } \\
\text { preference to instrumental assessment, considering the availability of } \\
\text { technical and human resources }\end{array}$ & Ila & C-EO \\
\hline Recommendation 5 & $\begin{array}{l}\text { Oral hygiene protocols that include brushing of the teeth/oral mucosa, } \\
\text { hydration and protection of the mouth (lips and mucous membranes) } \\
\text { should be instituted. In the case of severe dysphagia, it is reasonable to use } \\
\text { an antiseptic ( } 0.12 \% \text { chlorhexidine) twice a day to rinse the oral cavity }\end{array}$ & Ila & $B-R$ \\
\hline Recommendation 6 & $\begin{array}{l}\text { All stroke patients should be screened for malnutrition risk within } 48 \mathrm{~h} \text { of } \\
\text { hospitalization (ideally in the first } 24 \mathrm{~h} \text { ), using Nutritional Risk Screening } \\
2002 \text {, by a nurse, doctor or nutritionist. All patients at risk should be } \\
\text { referred to a nutritionist. The assessment and intervention of the } \\
\text { nutritionist must be carried out within a period of } 24 \mathrm{~h}\end{array}$ & I & C-EO \\
\hline Recommendation 7 & $\begin{array}{l}\text { Patients with impaired swallowing who are malnourished, or at risk of } \\
\text { malnutrition, should receive supplementary nutritional therapy through } \\
\text { an individualized (nutritional) plan developed and monitored by a } \\
\text { nutritionist in collaboration with a multidisciplinary team }\end{array}$ & I & C-EO \\
\hline Recommendation 10 & $\begin{array}{l}\text { When it is expected that enteral feeding should be needed over a month, } \\
\text { the insertion of a feeding tube through percutaneous endoscopic } \\
\text { gastrostomy will be beneficial }\end{array}$ & Ila & C-EO \\
\hline Recommendation 11 & $\begin{array}{l}\text { The presence of a nasogastric tube does not collide with therapeutic } \\
\text { intervention, therefore it should be started as early as possible }\end{array}$ & I & C-EO \\
\hline Recommendation 12 & $\begin{array}{l}\text { Behavioral intervention, including compensatory strategies (modification } \\
\text { of food consistencies and liquid viscosity, postural, and swallowing } \\
\text { techniques) and rehabilitation (muscle-strengthening exercises, resistance } \\
\text { or skill training), must be considered as a treatment component of } \\
\text { dysphagia in stroke patients }\end{array}$ & I & C-EO \\
\hline Recommendation 13 & $\begin{array}{l}\text { Before starting the behavioral intervention for the treatment of dysphagia, } \\
\text { it is recommended that all patients undergo a clinical evaluation of } \\
\text { swallowing (preferably instrumental). During treatment, and depending } \\
\text { on the clinical evolution, patients should be periodically reassessed }\end{array}$ & I & C-EO \\
\hline Recommendation 14 & $\begin{array}{l}\text { The systematic performance of different exercises and maneuvers, } \\
\text { provided for in an individualized therapeutic plan and adjusted to the } \\
\text { clinical condition, is recommended in stroke patients with dysphagia }\end{array}$ & I & C-EO \\
\hline
\end{tabular}


Table 3 (continued)

\begin{tabular}{|c|c|c|c|}
\hline & & $\begin{array}{l}\text { Class of } \\
\text { recommendation }\end{array}$ & $\begin{array}{l}\text { Level of } \\
\text { evidence }\end{array}$ \\
\hline Recommendation 15 & $\begin{array}{l}\text { It is reasonable to incorporate the principles of neuronal plasticity in the } \\
\text { strategies/intervention in the therapeutic plan of stroke patients with } \\
\text { dysphagia }\end{array}$ & Ila & C-EO \\
\hline Recommendation 16 & $\begin{array}{l}\text { Acupuncture can be considered an adjunctive treatment for dysphagia, } \\
\text { which must be performed by properly qualified professionals, specially } \\
\text { trained for these techniques }\end{array}$ & $\mathrm{Ilb}$ & C-LD \\
\hline Recommendation 17 & $\begin{array}{l}\text { The benefit of pharmacological treatment, pharyngeal electrical } \\
\text { stimulation, transcranial electrical stimulation and transcranial magnetic } \\
\text { stimulation is uncertain, so its use is not recommended }\end{array}$ & III - no benefit & C-LD \\
\hline Recommendation 18 & $\begin{array}{l}\text { The benefit of neuromuscular electrical stimulation is uncertain. As long as } \\
\text { there is no clinical contraindication, and despite the lack of high-quality } \\
\text { evidence to support its use, it may be reasonable to consider this strategy } \\
\text { as an adjunctive therapeutic option, under strict clinical evaluation and } \\
\text { monitoring }\end{array}$ & Illb & C-LD \\
\hline Recommendation 19 & $\begin{array}{l}\text { The increase in the viscosity of liquids reduces the risk of aspiration, } \\
\text { however, thickened liquids increase the risk of post-swallowing oral and } \\
\text { pharyngeal residue. Modification of food consistencies and the use of } \\
\text { thickeners should only be prescribed after clinical and/or instrumental } \\
\text { evaluation. The assessment should be repeated at regular intervals }\end{array}$ & I & C-EO \\
\hline Recommendation 20 & $\begin{array}{l}\text { The use of thickeners decreases fluid intake. Thickened fluids should be } \\
\text { used in people with dysphagia for fluids. However, fluid intake must be } \\
\text { closely monitored due to insufficient intake risk }\end{array}$ & I & C-EO \\
\hline Recommendation 21 & $\begin{array}{l}\text { Even with changes in food consistencies and fluid viscosity, patients with } \\
\text { dysphagia are at increased risk of malnutrition, dehydration and } \\
\text { pneumonia. Therefore, they must be closely monitored for these } \\
\text { complications }\end{array}$ & I & C-EO \\
\hline
\end{tabular}

ally, all patients should be evaluated with reference tests; however, there are several limitations: not all patients can undergo an invasive examination, nor do all hospitals have trained professionals available $24 \mathrm{~h}$ a day to perform them and not all hospitals have the necessary equipment [15]. As a result, instrumental assessment is not accessible to all stroke patients in the acute phase. Therefore, dysphagia screening is recommended in several clinical guidelines for all patients admitted with stroke, as early as clinically possible, before the administration of any food, liquid or medication [15-22]. Of all these recommendations, only one sets a time interval of up to $4 \mathrm{~h}$ after admission [20]. All the others indicate as early as possible and as soon as the patient's level of consciousness allows it. The supporting evidence results are essentially from observational studies. In fact, a systematic review published in 2018, which sought to identify RCTs that studied the effect of dysphagia screening protocols on the incidence of pneumonia, death or dependence after stroke, only 3 studies were identified, and the results did not determine any effect on these outcomes [23]. Another systematic review of observational and quasi-experimental studies, which included 12 studies with more than 87,000 participants, suggests that early screening for dysphagia reduces the incidence of pneumonia after stroke [24]. According to the authors, the heterogeneity of the methods prevented the quantitative analysis of the results. Evidence supporting the international recommendations comes essentially from studies included in this latest review [24]. An observational study published later and not included in this review [25], with a sample of 3,309 participants, who sought to assess the impact of delay in screening for dysphagia in stroke patients, regarding its performance within $4 \mathrm{~h}$ after admission, concluded that the delay is harmful, suggesting that this may be associated with the delay in nutrition and the use of inappropriate feeding techniques, which enhances aspiration. This study reinforces the existing evidence, thus justifying the 
level of recommendation proposed for the first recommendation. Thus, screening is the first step to identifying patients at risk and in need of a more comprehensive clinical or instrumental assessment [17-19, 26, 27]. Silent aspiration is an added challenge for health professionals, and the range of dysphagia screening tools available does not adequately address this dimension of dysphagia. Most of them consider open signs of aspiration but do not consider, for example, the capacity for reflex coughing, which reflects the degree of damage to the laryngeal sensorimotor response $[28,29]$, thus reinforcing the need for clinical evaluation not only for patients with positive screening, but also for those with other risk factors. The American Heart Association/American Stroke Association (AHA/ ASA) recommend, in a moderate grade, that all patients must undergo instrumental assessment subject to the availability of human and technical resources [16, 22], arguing that instrumental assessment allows visualizing the physiology of swallowing and determine the presence or absence of aspiration, which is necessary for the definition of an adequate therapeutic plan. Other guidelines suggest instrumental assessment following an inconclusive clinical assessment/suggestive of aspiration or in enteral-fed persons $[17,18]$. Another guideline, recognizing the usefulness of performing clinical assessment, recommends that preference be given to instrumental assessment [21]. The recommendation essentially emerges from the awareness of the limited human and technical resources for prioritizing instrumental assessment. Regarding the professional most qualified to perform the screening, part of the international guidelines recommend that it should be performed by an SLP $[16,17,22]$ or another properly trained professional, which they do not specify $[18,19]$. One of the recommendations does not refer to the professional who should perform the screening [21]. Evidence suggests that screening by nurses is effective in an important set of outcomes in stroke patients, namely in reducing the incidence of pneumonia $[30,31]$. In this context, there is a relatively established consensus that screening for dysphagia in stroke patients should be carried out by nurses as soon as possible, so that patients are not kept nil by mouth for an unnecessary time, considering that an SLP is not available $24 \mathrm{~h}$ /day in hospitals [31, 32].

\subsection{Certainty of Evidence of Effects}

Despite a wide international consensus amongst experts for early screening, most of the available data are observational and quasi-experimental studies (limited data) and are not conclusive. Nevertheless, the available evidence suggests higher benefit in screening versus no screening and early screening versus delayed screening. The remaining recommendations stand mostly on experts' opinions.

\subsection{Summary of Delphi Panel's Results}

For the first recommendation, $100 \%$ of the total consensus was obtained in the first round. Concerning instrumental assessment, to note that one of the experts stated that it should be clear that only SLPs can perform the clinical assessment and subsequent intervention, adding that the instrumental assessment should only be performed in case of doubtful clinical assessment. Regarding professionals fit to screen stroke patients, one of the experts partially agreed with the recommendation, stating that SLPs should perform clinical assessment and not screening. Screening should be performed by properly trained nurses or physicians. Two other experts suggested including the physician, noting that, in certain clinical contexts, the physician also performs screening. The recommendation built from the experts' consensus was classified as strong given the need to, following the recommendation regarding early screening, ensure that clear guidance is provided.

\subsection{Conclusions and Research Need for this}

Recommendation

Unequivocal evidence on the impact of early dysphagia screening in acute stroke patient's outcomes is insufficient. RCTs are needed to determine the effect on reducing pneumonia rates and death and on the improvement of functional outcomes. These findings and suggestions for future trials do not mean that screening should not be performed. Strong consensus in both national and international experts is found on this subject. For dysphagia, more specifically for the identification of aspiration, instrumental assessment has been considered the gold standard. However, it is essential to further alignment in defining the focus of research on the assessment of dysphagia, with significance for clinical practice. Limited resources for the realization of instrumental assessment could even be exceeded in the context of clinical trials through funding, but it would not mimic reality in clinical settings, limiting the applicability of these results.

\section{Clinical Question}

What tools are available to screen dysphagia in acute stroke patients? 


\subsection{Recommendation Statement}

It is recommended that dysphagia screening protocols use validated screening instruments. The Gugging Swallowing Screen (GUSS), due to its psychometric properties, seems to be an adequate instrument.

\section{COR IIa}

\section{LOE C-EO}

\subsection{Summary of Evidence}

There is a consensus on the need to implement dysphagia screening protocols in acute stroke patients; however, not all stroke units use dysphagia screening protocols, and in Portugal, there is evidence that nurses use water tests and different items for informal dysphagia screening [33]. Informal detection, despite having high specificity, has low sensitivity, which means that a significant percentage of dysphagic patients will not be identified, translating into an increased risk of complications [34]. Water tests and pulse oximetry assessment, traditionally used to identify aspiration, are often used as dysphagia screening strategies, whereas for water tests, the result for sensitivity is less than $80 \%$ [35] and concerning pulse oximetry, the existing evidence cannot prove the existence of an association between oxygen desaturation and aspiration, thus not validating its use in detecting aspiration [36]. These findings reinforce the need to implement dysphagia screening protocols to identify patients at risk who need subsequent clinical evaluation [33]. For screening, no specific tool has been recommended so far in most clinical guidelines probably due to the difficulty in developing instruments with the ideal psychometric properties, i.e., adequate sensitivity, specificity and predictive strength that allow to detect dysphagia and aspiration when used by any health care professional $[17,19,20,26]$. A systematic review identified three dysphagia screening tools for stroke patients, validated against the gold standard [37]. Given its psychometric properties and dietary recommendations, the GUSS seems to be the most appropriate tool available so far [37] and is referred to in two clinical guidelines [18, 27]. It is a two-part screen, comprising in the first part the test of indirect swallowing items (level of consciousness, saliva swallowing, voice changes, drooling and voluntary cough) that, if successfully achieved, leads to the part where swallowing items are tested with different consistencies and volumes of liquids, semisolid and solid textures, in a series of sequential subtests [38].

\subsection{Certainty of Evidence of Effects}

Evidence on the use of the GUSS is increasing, and a systematic review [39] showed in a pooled analysis a sen- sitivity of 0.97 and specificity of 0.67 , concluding that the screening performed by nurses using the GUSS reduces the time for screening and the incidence of pneumonia making it a valid and sensitive tool for dysphagia screening. Thus, it is expected that the benefit of using a dysphagia screening tool in patients with stroke is higher than not using one.

\subsection{Summary of Delphi Panel's Results}

A very strong consensus was obtained for this recommendation. Two experts stated some reservations about the use of the GUSS, noting that the instrument has some gaps that would be important to fill, adding that from all of the available screening tools, this one seems to be the most suitable.

\subsection{Conclusions and Research Need for this}

\section{Recommendation}

A single screening tool may not be adequate to all clinical settings due to organizational, clinical or structural issues and availability of resources, that is why concerns in research should focus on substantiating the effectiveness of the tools already developed, to strengthen the results of validity and reliability, as well as assess the impact on outcomes in stroke patients. Different tools have been developed with similar psychometric properties; spending resources on research to develop new screening tools does not seem to bring better outcomes for patients.

\section{Clinical Question}

Does the introduction of oral hygiene protocols, compared to standard oral hygiene care, decrease mortality and morbidity or improve health outcomes in stroke patients with dysphagia?

\subsection{Recommendation Statement}

It is recommended to implement oral hygiene protocols that include brushing of the teeth/oral mucosa, hydration and protection of the mouth (lips and mucous membranes). In the case of severe dysphagia, it is reasonable to use an antiseptic ( $0.12 \%$ chlorhexidine) twice a day to rinse the oral cavity.

\section{COR IIa \\ LOE B-R}

\subsection{Summary of Evidence}

Stroke patients are at increased risk of oral colonization by respiratory pathogens, which is associated with adverse respiratory events $[40,41]$ and poorer quality of life [42]. Furthermore, they have a worse clinical oral 
health status in several parameters: tooth loss, number of caries, periodontal status and oral hygiene $[43,44]$. On the other hand, there is also evidence of the association between dysphagia and an increased risk of pneumonia $[45,46]$ which, in turn, is associated with an increased time of hospitalization, worse functional outcomes and increased risk of mortality [47]. Despite this, the available evidence does not allow for an independent association between dysphagia and aspiration pneumonia, as it is multifactorial. There is evidence to suggest that patients with a nasogastric tube (NGT), whose presence promotes colonization of the oropharynx, patients with severe mobility impairment and patients with an altered state of consciousness are at greater risk of developing aspiration pneumonia $[4,48]$. On the other hand, a study developed by Kalra et al. [49] concludes that the early use of an NGT in stroke patients not orally fed does not increase the incidence of pneumonia, mortality or worse functional outcomes. In addition, free water intake, under specific conditions, in patients with dysphagia after stroke, does not increase the incidence of aspiration pneumonia, thus reinforcing its multifactorial etiology [50].

In this context, international guidelines emphasize the importance of implementing oral hygiene programs, that show explicitly the frequency of mouth care, devices and products to be used, stressing the importance of these protocols for pneumonia reduction [16-20, 22]. Oral care after every meal and bedtime is recommended [19], or twice a day $[51,52]$, and it is considered reasonable to perform it with a rinse with chlorhexidine to reduce the risk of pneumonia, dental plaque and bleeding gums [16, 20, 51, 52].

The use of chlorhexidine in a rinse solution or gel in oral hygiene care has been shown to be effective in reducing ventilator-associated pneumonia in critically ill patients [53]; however, there is insufficient evidence to establish this relationship in stroke patients. Updated evidence shows that adding chlorhexidine to oral care reduced dental plaque and gingival bleeding [54], and the incidence of aspiration pneumonia [55]. The implementation of oral hygiene programs with increased frequency and intensity suggests a significant improvement in oral health [56], and its implementation associated with the use of other oral solutions for washing and rinsing also suggests improved oral health and reduced incidence of aspiration pneumonia [57].

The need for training and education for health professionals on the assessment, provision and importance of oral care in preventing pneumonia is acknowledged [20]. The intervention of professionals in oral hygiene seems to improve the knowledge and attitudes of professionals

Best Practice in Dysphagia after Stroke and reduce the incidence of pneumonia [58]. Despite that, oral care is overlooked in clinical practice which reflects the professionals' lack of knowledge in oral care. Contributing to it is the lack of evidence and specific guidelines for stroke patients.

\subsection{Certainty of Evidence of Effects}

Evidence suggests that a superior benefit resulting from the implementation of oral hygiene protocols that include brushing of the teeth/oral mucosa, hydration and protection of the mouth (lips and mucous membranes) in stroke patients will be expected compared to its nonimplementation $[59,60]$ and that the use $0.12 \%$ chlorhexidine has a favorable effect on the incidence of respiratory complications in patients with severe dysphagia [55].

\subsection{Summary of Delphi Panel's Results}

A strong consensus was obtained for this recommendation. One of the experts partially agreed on this recommendation stating that the recommendation should include the frequency of oral care. Another expert raised some concerns on the effect of chlorhexidine on the swallowing ability, justifying a partial agreement to the recommendation. For this reason, further search was made of RCTs on the effect of chlorhexidine on swallowing ability, with no results. The most frequently reported adverse effects reported are changes in taste, changes in the oral mucosa (discomfort/pain, irritation, slight desquamation and ulceration/erosion) and burning sensation in the mouth and/or tongue [61].

\subsection{Conclusions and Research Need for this}

\section{Recommendation}

Systematic oral care may reduce the incidence of pneumonia; however, further studies to make this relationship unambiguous are needed [62]. The analysis of the evidence found highlights the need to produce evidence, with more robust designs, to identify the oral hygiene protocol that produces the best results improving oral health, quality of life and reducing the incidence of complications.

The need for education and training on oral care for health professionals, especially nurses, and caregivers is emphasized.

\section{Clinical Question}

Does systematic screening for nutritional risk, compared to standard care, in stroke patients with dysphagia decrease mortality and morbidity or improve health outcomes in stroke patients? 


\subsection{Recommendation Statement(s)}

It is recommended to screen all stroke patients for malnutrition risk within $48 \mathrm{~h}$ of hospitalization (ideally in the first $24 \mathrm{~h}$ ), using Nutritional Risk Screening 2002 (NRS 2002), by a nurse, doctor or nutritionist. It is recommended to refer patients at risk to a nutritionist. It is recommended that the assessment and intervention of the nutritionist be carried out within a period of $24 \mathrm{~h}$.

\section{COR I}

\section{LOE C-EO}

It is recommended that patients with impaired swallowing who are malnourished or at risk of malnutrition receive supplementary nutritional therapy through an individualized (nutritional) plan developed and monitored by a nutritionist in collaboration with the multidisciplinary team.

\section{COR I}

\section{LOE C-EO}

\subsection{Summary of Evidence}

There is evidence of an association between dysphagia and increased dehydration in the first 7 days after stroke [5], as well as an increase in malnutrition $[5,6]$. These complications result in increased length of stay, greater likelihood of institutionalization after discharge, increased mortality and worse functional outcomes $[7,45,63]$. Furthermore, the nutritional deficit is also associated with a worse quality of life in stroke patients [64]. It is in this context that different international guidelines recommend nutritional risk assessment to all patients after a stroke, however with differences in recommendations. The European Society for Clinical Nutrition and Metabolism (ESPEN) [21] recommends the use of the Malnutrition Universal Screening Toll (MUST) based on the validation study of MUST for stroke patients [65]. In Portugal, guidelines recommend the use of Nutritional Risk Screening 2002 (NRS 2002) [66].

Recognizing the increased risk of malnutrition in stroke patients with dysphagia, nutritional risk assessment is recommended to all patients within the first 48 $\mathrm{h}$ of hospitalization (ideally during the first $24 \mathrm{~h}$ ), using the NRS 2002 [66]. Screening can be performed by a nurse, a doctor or a nutritionist, and patients at risk should be referred for nutritional assessment and intervention within $72 \mathrm{~h}$ after admission [66]. Patients must be weekly reassessed [20] and for patients at nutritional risk, individualized nutritional support is recommended [67].

\subsection{Certainty of Evidence of Effects}

Nutritional support does not reduce adverse events or show any effect in reducing mortality [67]; however, the recommendation level is strong, supported essentially by expert opinion, considering that the benefit of the intervention greatly outweighs the risk.

\subsection{Summary of Delphi Panel's Results}

The first version of the recommendations on systematic nutritional screening proposed the MUST as a screening tool, based on the ESPEN recommendations, a proposal that was rejected by several experts, referring to national guidelines. The recommendation was then revised, and in a second round received full agreement from all experts.

\subsection{Conclusions and Research Need for this}

\section{Recommendation}

The difference in the screening tool's recommendation may be related to the fact that national guidelines are not specifically aimed at stroke patients. Nevertheless, wide consensus exists on the need to screen stroke patients and provide individualized nutritional therapy developed and monitored by a nutritionist. Research is needed to determine the effects of nutrition support on death, dependency and other health-related outcomes.

\section{Clinical Question}

Does NGT feeding in patients with severe dysphagia, compared to other enteric feeding strategies, decrease mortality and morbidity or improve health outcomes in stroke patients?

\subsection{Recommendation Statement(s)}

It is recommended that enteral feeding starts as soon as clinically possible, after a stroke, in patients with severe dysphagia.

\section{COR I}

\section{LOE B-R}

NGT can be useful for short periods (up to 1 month) for nutritional support in patients who do not swallow safely.

\section{COR IIa}

\section{LOE C-EO}

When it is expected that enteral feeding should be needed over a month, the insertion of a feeding tube through percutaneous endoscopic gastrostomy (PEG) can be beneficial.

\section{COR IIa \\ LOE C-EO}


The presence of NGT does not collide with therapeutic intervention, therefore it is recommended to start as early as possible.

\section{COR I}

\section{LOE C-EO}

\subsection{Summary of Evidence}

Malnutrition in stroke patients can have a severe impact as it enhances worse functional results and worse quality of life [68], putting at risk the rehabilitation process. Malnutrition risk is also an independent predictor of mortality, longer hospital stay and hospitalization costs [65]. Enteric feeding is, in situations of severe dysphagia, the only way to ensure nutritional and hydration intake, as well as allowing the administration of medication [69]. However, its use is not without risks, with mechanical complications, such as nasal trauma, displacement, poor positioning and obstruction [70], gastrointestinal complications [71], metabolic disorders [72], resulting in a worse quality of life [73]. Different international guidelines recommend as soon as possible enteral nutrition by NGT in patients who, after a stroke, do not swallow safely, with recommendations varying between the first $24 \mathrm{~h}$ [18], the first 3 days $[17,19,21]$ or the first 7 days $[16,22$, 27]. The Australian recommendations only indicate, with a weak level of recommendation, that NGT enteral feeding is the preferred short-term method of feeding stroke patients [20].

The early placement of an NGT for feeding is justified by the enhanced nutritional risk, thus allowing adequate nutrition and hydration [21]. Wirth et al. [27] alert that these patients have a higher risk of aspiration and aspiration pneumonia that is not prevented with the placement of an NGT, so the risk of aspiration cannot be the reason for its placement. It should be noted that the evidence regarding the increased risk of pneumonia associated with NGT placement is contradictory [74].

Procedures for verifying the correct positioning and fixation of the NGT are recommended, considering the risks of displacement and accidental removal, issues with a significant impact on nutrient supply and a common problem in clinical practice [21]. Therefore, the use of small caliber tubes $(8 \mathrm{CH})$ is recommended to minimize the risk of ulceration; the verification of the correct placement of the NGT should be performed through Rx or aspiration of gastric contents for $\mathrm{pH}$ measurement and, for fixation, the use of a nasal loop/bridle is advised [21,27].

If it is expected that the patient will not be able to restart oral feeding for a period longer than 2-4 weeks, it is recommended to place a PEG $[14,16,21,22]$. In the deci- sion to place a tube for enteral feeding by gastrostomy, in stroke patients with an unfavorable prognosis, ethical issues and early manifestation of a will must be specially attended, and, when in doubt, enteric feeding by NGT should be considered as the most appropriate, given the reversibility of the procedure [21].

Therapeutic intervention for dysphagia should start as early as possible, considering that the presence of an NGT does not aggravate dysphagia [75] and does not interfere with swallowing training [27]. In fact, the presence of an NGT interferes with the movement of the hyoid bone during swallowing, but that this movement is re-established after removal of the tube [76].

\subsection{Certainty of Evidence of Effects}

The Feed or Ordinary Diet (FOOD) trial was a welldesigned multicentric RCT that aimed to evaluate feeding policies in stroke patients, that was developed in 131 hospitals in 18 countries, with 5,033 stroke patients [77] and is the supporting evidence of most international guidelines. More recent evidence suggests that in the comparison between enteral feeding by NGT versus PEG, PEG is associated with fewer treatment failures, lower incidence of gastrointestinal bleeding, higher feeding delivery and albumin concentration [78]. No differences in death or dependency were found.

\subsection{Summary of Delphi Panel's Results}

The consultation of the experts revealed high levels of agreement, although it was necessary to reformulate two of the recommendations. The first regarding the time to start enteral feeding (the first draft had a time limit of 72 $\mathrm{h}$ ), with $72 \mathrm{~h}$ being considered an excessively long period by five of the experts. It was agreed that it should be started as soon as clinically possible. The second, regarding the start of therapeutic intervention, in which five of the experts did not agree with the initial wording that stated that the presence of an NGT does not worsen dysphagia, agreeing, however, that the therapeutic intervention should be started as early as possible. For this reason, recommendations were revised to incorporate the experts' suggestions.

\subsection{Conclusions and Research Need for this}

Recommendation

Despite the lack of robust evidence, adequate nutrition and hydration must be ensured in stroke patients with severe dysphagia considering the nefarious impact of dehydration and malnutrition on the rehabilitation process. This is an area that needs further investigation, recogniz- 
ing the ethical and logistical challenges that studies with vulnerable populations represent, which translates into the difficulty of conducting truly randomized clinical trials. The possibility of predicting the duration of dysphagia is crucial for the decision-making process regarding the route for feeding. Research must address this issue.

\section{Clinical Question}

Which therapeutic interventions have the most significant results in the recovery of swallowing function and airway safety?

\subsection{Recommendation Statement(s)}

It is recommended to consider behavioral intervention, including compensatory strategies (modification of food consistencies and liquid viscosity, postural and swallowing techniques) and rehabilitation (muscle-strengthening exercises, resistance or skills training), as a treatment component of dysphagia in stroke patients.

\section{COR I}

\section{LOE C-EO}

Before starting the behavioral intervention for the treatment of dysphagia, it is recommended that all patients undergo a clinical evaluation of swallowing (preferably instrumental). During treatment, and depending on the clinical evolution, it is recommended to periodically reassess patients.

\section{COR I}

\section{LOE C-EO}

The systematic performance of different exercises and maneuvers, provided for in an individualized therapeutic plan and adjusted to the clinical condition, is recommended in stroke patients with dysphagia.

\section{COR I}

\section{LOE C-EO}

It is reasonable to incorporate the principles of neuronal plasticity in the strategies/interventions in the therapeutic plan of stroke patients with dysphagia.

\section{COR IIa}

\section{LOE C-EO}

Acupuncture may be considered an adjunctive treatment for dysphagia, which must be performed by properly qualified professionals, specially trained for these techniques.

\section{COR IIb}

\section{LOE C-LD}

The benefit of pharmacological treatment, pharyngeal electrical stimulation, transcranial electrical stimulation and transcranial magnetic stimulation is uncertain, so its use is not recommended.

\section{COR III - no benefit \\ LOE C-LD}

The benefit of neuromuscular electrical stimulation is uncertain. As long as there is no clinical contraindication, and despite the lack of high-quality evidence to support its use, it may be reasonable to consider this strategy as an adjunctive therapeutic option, under strict clinical evaluation and monitoring.

\section{COR IIb \\ LOE C-LD}

\subsection{Summary of Evidence}

Defining recommendations for the therapeutic approach to patients with dysphagia after stroke is, most likely, one of the biggest challenges in this context. The range of interventions available is vast and includes behavioral interventions that comprise compensatory strategies (postural and swallowing techniques and dietary modifications) and rehabilitation strategies (strengthening, resistance and skill training) [79]. In addition to these interventions, there are other rehabilitation strategies, such as noninvasive neurostimulation techniques: repetitive transcranial magnetic stimulation (RTMS), transcranial direct current stimulation (TDCS), neuromuscular electrical stimulation (NMES) and pharyngeal electrical stimulation (PES) [80]; a physical stimulation (tactile, thermal and sour); pharmacological strategies [79] and acupuncture [81]. The distinction between the different compensatory and rehabilitation strategies becomes particularly relevant as each of them has specific objectives that are not mutually exclusive: the compensatory strategies aim to ensure adequate and safe hydration and nutrition, preventing complications, and rehabilitation strategies have as main objective the recovery of the swallowing function [14].

The analysis of international clinical guidelines reflects the difficulty to summarize the evidence, including contradictory recommendations regarding the same therapeutic strategy. As an example, the clinical guidelines of the AHA/ASA recommend the nonuse of NMES because its benefits are uncertain, supporting this recommendation in the systematic review produced by the Cochrane Collaboration [78]. On the other hand, ESPEN's recommendations are to recommend its use, alone or, preferably, with behavioral intervention [21]. It is important to note that, in addition to these two guidelines, only in the Australian clinical guidelines have recommendations on the use of NMES, recommending its use only by experienced clinicians, been applied according to parameters established in the research context and with a weak 
level of recommendation [20]. Both the ESPEN recommendations [21] and the Stroke Foundation [20] recommendations support its recommendation in a systematic review published in 2016 [82] which suggests that, despite the limited number of available studies, NMES appears to be more effective in the short term in post-stroke dysphagia. However, it also states that the existing evidence does not allow to determine whether isolated NMES is superior to swallowing therapy.

Nevertheless, in stroke patients with dysphagia, it is strongly recommended to define an individualized therapeutic plan, with early use of behavioral intervention, which should include rehabilitation and compensatory strategies such as swallowing exercises, environmental/ postural changes, education on safe swallowing and modification of food consistencies $[17,20,21,22]$. As the instrumental assessment is the reference test for the assessment of swallowing, allowing the visualization of the physiology of swallowing and determining the pathophysiological and structural causes of dysphagia [14, 83], such information is essential for the design of an appropriate and effective therapeutic plan.

Despite the scant research carried out focusing on rehabilitation in the first 2 weeks after stroke [84, 85], early start of rehabilitation, $72 \mathrm{~h}$ after the onset of symptoms, has a greater impact on the recovery of swallowing function, as well as on the reduction of the incidence of respiratory complications [86]. Despite not being entirely clear what the maximum neuroplasticity period is, it is particularly increased right after the stroke, during which the dynamic response of the brain to the injury is intensified and rehabilitation can be particularly effective [84]. Therefore, a therapeutic intervention must be systematic, frequent and periodically reassessed $[17,20,21]$.

Concerning rehabilitations strategies, none of the interventions has a significant impact on death or dependence [89]. Acupuncture, behavioral intervention and RTMS may be effective, namely in reducing the proportion of patients with dysphagia, improving swallowing capacity and reducing penetration/aspiration [79, 81]. However, the results may be due to chance, given the reversibility of the clinical condition. Furthermore, these results are supported by low-quality evidence. The AHA/ ASA recommend that behavioral interventions and acupuncture may be considered in dysphagia treatment [22].

Analysis on the effectiveness of noninvasive neurostimulation techniques in patients with post-stroke dysphagia suggests benefits in the use of RTMS, TDCS and NMES, however with superior benefits for RTMS [80]. Recommendations made in different international guide-

Best Practice in Dysphagia after Stroke lines are contradictory concerning pharmacological treatment $[21,22]$, therefore preventing any recommendation. Caution is recommended in interpreting these results given the high heterogeneity of the studies, thus not allowing to make recommendations on the more effective protocols.

More recent evidence suggests a reduction in penetration/aspiration with behavioral intervention [87] and NMES [88]. Improvement of swallowing functions was identified with behavioral intervention [87], NMES [88], RTMS [89] and acupuncture [90].

\subsection{Certainty of Evidence of Effects}

Most studies have high heterogeneity in intervention and outcome measurements. The quality of the evidence produced is moderate to low, mainly due to the lack of true randomization considering the difficulty of concealing the participant's allocation and/or the intervention, which, in studies of this nature, proves to be a challenge. The apparent contradictions identified in the different clinical guidelines result from a set of factors that have highlighted the constraints of research in this area: the lack of uniformity in the assessment of dysphagia and the diversity of existing measurement instruments (whether clinical or instrumental); the lack of uniformity in measuring the results of interventions; the variability of available treatments and possible combinations, with no standardized interventions; the low methodological quality and high risk of bias in most published studies; the high probability of reversibility of the clinical condition (a significant percentage of patients spontaneously recover the ability to swallow), which means that the positive results of the studies may be due to chance [79-81]. Therefore, the estimation of the true effect/efficacy of interventions is limited.

\subsection{Summary of Delphi Panel's Results}

From the first Delphi round, the disagreement of the experts is worth noting regarding the uncertainty in the use of the NMES, which led to the elaboration of a specific recommendation. The first draft of recommendations was to advise against the use of NMES, due to the lack of high-quality evidence to support it.

\subsection{Conclusions and Research Need for this}

Recommendation

Defining a therapeutic intervention for dysphagic stroke patients is, certainly, one of the major difficulties for health care providers. There is not sufficient evidence to determine whether improvement in swallowing func- 
tion is due to the spontaneous recovery or due to the treatment, which enhances the need for systematic assessment and management of these patients. For research, constraints already identified must be addressed: the need for a clear definition of the time elapsed after the stroke at the time of recruitment, seeking to reach large samples, ideally from different centers; in the absence of proven intervention, participants in the control group should only receive standard care; the use of similar research methods thus allowing comparison, and the standardization of results and outcome measures. For outcome measurements and, additionally, functional outcomes (death and dependence), the proportion of patients who develop respiratory infection or pneumonia or show signs of aspiration and results relevant to the health economy such as length of stay, as well as the quality of life, should be assessed.

\section{Clinical Question}

Does modifying the consistency of food and liquids compared to standard feeding decrease mortality and morbidity or improve health outcomes in stroke patients?

\subsection{Recommendation Statement(s)}

The increase in the viscosity of liquids reduces the risk of aspiration; however, thickened liquids increase the risk of post-swallowing oral and pharyngeal residue. It is recommended that modification of food consistencies and the use of thickeners only be prescribed after clinical and/ or instrumental evaluation. It is recommended to repeat assessments at regular intervals.

\section{COR I}

\section{LOE C-EO}

The use of thickeners decreases fluid intake. It is recommended to thicken fluids in patients with dysphagia for fluids. However, it is recommended to closely monitor fluid intake due to insufficient intake risk.

\section{COR I}

\section{LOE C-EO}

Even with changes in food consistencies and fluid viscosity, patients with dysphagia are at increased risk of malnutrition, dehydration and pneumonia. Therefore, it is recommended that they are closely monitored for these complications.

\section{COR I \\ LOE C-EO}

\subsection{Summary of Evidence}

The modification of food consistencies and thickening of liquids is probably the compensatory strategy mostly used in patients with dysphagia; however, the recommendation for its use is much more based on a consensus of good practice than on evidence from research [91-94]. Evidence on the safety and effectiveness of using strategies to increase the viscosity of liquids is scarce and, in the case of changes in food consistencies and use of mixed consistencies, it is residual [93].

Increasing the bolus viscosity increases the safety of swallowing, and the use of thickeners decreases penetration/aspiration; however, it increases the post-swallowing residue which can result in post-swallowing airway invasion [93]. In addition, it has an impact on the physiology of swallowing with an increase in the tongue pressure pattern, but without impact on airway involvement and with controversial effect on oral and pharyngeal transit times, beginning of the opening of the upper esophageal sphincter and bolus speed [94]. The use of thickened liquids results in an increased risk of dehydration [95], a higher prevalence of silent aspiration and worse quality of life [96]. The use of thickeners may nullify the effect of drugs and the likelihood of error in the administration of drug therapy is much higher in dysphagic patients [97]; however, there is insufficient evidence or consensus to formulate recommendations.

Patients report the experience of using thickeners as unpleasant, and this displeasure may negatively influence adherence to this therapeutic strategy, hydration status and quality of life [98]. They also point out the lack of sensorial appeal as an important basis for the displeasure in relation to dietary changes and thickening of liquids, in addition to the fact that the involvement and understanding of the reasons for the prescription are low, leading to uncertainties about the treatment. It should be noted that it is recommended that, based on an individual assessment and decision and regular monitoring, additional access to nonthickened water be given to patients with liquid aspiration [21]. In fact, the implementation of protocols for the intake of unthickened water under specific conditions may improve the quality of life [99]; however, there are barriers to its implementation, namely the nurses' lack of expertise in performing oral hygiene, lack of adherence to the rules of the protocols, perception of the increased workload for nurses and an established culture for the use of thickeners [99].

\subsection{Certainty of Evidence of Effects}

Despite the strong consensus amongst experts, nationally and internationally, it must be stated that there are limited data to support any of the recommendations. In fact, dietary modifications and fluid thickening are costly 
interventions without empirical support for their use, and there is growing evidence that they do more harm than good, for example, dehydration.

\subsection{Summary of Delphi Panel's Results}

All recommendations achieved full agreement from all experts in the first round.

\subsection{Conclusions and Research Need for this}

Recommendation

The use of dietary and fluid modifications in dysphagic patients depends on a thorough assessment of the patient's ability to swallow, considering the increased risk of dehydration and post-swallowing residue. The main limitations on research in this field are the small sample size, including participants with multiple clinical conditions, heterogeneity in the methods and measurement of results, as well as the lack of uniformity in the definition and terminology of viscosity levels, which must be addressed in future research. It is also necessary to deepen the research regarding the administration of medication in dysphagic patients.

\section{Conclusion}

These recommendations aimed to gather the most recent evidence and highlight points of convergence in the existing recommendations for assessment and management of dysphagia in stroke patients. The various studies included in these recommendations make it possible to understand the impact that dysphagia has on a patient from a clinical point of view. Respiratory complications have been the main focus of research worldwide and the commitment to nutrition, hydration and quality of life of the person and family has not deserved the same attention. There is little evidence about the quality of life of stroke patients with dysphagia and their families.

These clinical recommendations are an overview of the most recent evidence translated into clinically relevant statements. In implementing recommendations at the local level, health professionals should identify facilitators and barriers to evidence-based practice within their own contexts and determine the best strategies to address local needs. Where change is needed, initial and continuing training on all recommendations is essential and relevant.

\section{What this Work Adds to Current Practice}

To our knowledge, these are the first specific national recommendations for the assessment and management of dysphagia in stroke patients and will provide health care practitioners with evidence-based guidelines translated into clinically relevant statements to assist in the decision-making process.

\section{Limitations}

Most of the evidence found is from moderate to low quality and denotes the need for further research to identify the best therapeutic options for these patients.

Patients were not involved in the elaboration of these recommendations, and this is acknowledged as one of its limitations. Due to the low quality of evidence, most recommendations are grounded in experts' opinions. This might result in potential issues related to the diversity of clinical contexts and practices limiting their applicability. Recommendations should be reviewed within 5 years or if relevant evidence is identified before that period.

\section{Statement of Ethics}

This paper corresponds to a systematic literature review followed by a multidisciplinary panel of experts. All these experts were informed about the study aims, and participation was voluntary and anonymous. The consent was assumed after the participation of experts.

\section{Conflict of Interest Statement}

The authors declare no financial or other conflicts of interest.

\section{Funding Sources}

No funds, nor any sort of payment, were received. CEISUC/ CIBB is funded by national funds through FCT - Foundation for Science and Technology, I.P., under the Multiannual Financing of R\&D Units 2020-2023.

\section{Author Contributions}

Conceptualization: Isabel J. Oliveira, Germano R. Couto, Pedro L. Ferreira. Data curation: Isabel J. Oliveira, Rosa V. Santos, Ana M. Campolargo, Cláudia Lima. Formal analysis: Isabel J. Oliveira, Rosa V. Santos, Ana M. Campolargo, Cláudia Lima. Investigation: Isabel J. Oliveira, Rosa V. Santos, Ana M. Campolargo, Cláudia Lima. Methodology: Isabel J. Oliveira, Germano R. Couto, Pedro L. Ferreira. Project administration: Isabel J. Oliveira, Germano R. 
Couto, Pedro L. Ferreira. Supervision: Germano R. Couto, Pedro L. Ferreira. Validation: Germano R. Couto, Pedro L. Ferreira. Writing - original draft: Isabel J. Oliveira, Germano R. Couto, Pedro L. Ferreira. Writing - review and editing: Isabel J. Oliveira, Germano R. Couto, Rosa V. Santos, Ana M. Campolargo, Cláudia Lima, Pedro L. Ferreira.

\section{Data Availability Statement}

All data generated or analyzed are included in this article in the supplementary material files. Further enquiries can be directed to the corresponding author.

\section{References}

1 World Health Organization. The top 10 causes of death [Internet]. Geneva: World Health Organization; 2021 [cited 2018 May 24]. Available from: https://www.who.int/en/ news-room/fact-sheets/detail/the-top-10causes-of-death.

2 Johnson CO, Nguyen M, Roth GA, Nichols E, Alam T, Abate D, et al.; GBD 2016 Stroke Collaborators. Global, regional, and national burden of stroke, 1990-2016: a systematic analysis for the Global Burden of Disease Study 2016. Lancet Neurol. 2019 May;18(5):439-58.

3 Takizawa C, Gemmell E, Kenworthy J, Speyer R. A systematic review of the prevalence of oropharyngeal dysphagia in stroke, Parkinson's disease, Alzheimer's disease, head injury, and pneumonia. Dysphagia. 2016 Jun;31(3):434-41.

4 Brogan E, Langdon C, Brookes K, Budgeon C, Blacker D. Dysphagia and factors associated with respiratory infections in the first week post stroke. Neuroepidemiology. 2014;43(2):140-4.

5 Crary MA, Humphrey JL, Carnaby-Mann G, Sambandam R, Miller L, Silliman S. Dysphagia, nutrition, and hydration in ischemic stroke patients at admission and discharge from acute care. Dysphagia. 2013 Mar;28(1):69-76.

6 Chen N, Li Y, Fang J, Lu Q, He L. Risk factors for malnutrition in stroke patients: a metaanalysis. Clin Nutr. 2019 Feb;38(1):127-35.

7 Attrill S, White S, Murray J, Hammond S, Doeltgen S. Impact of oropharyngeal dysphagia on healthcare cost and length of stay in hospital: a systematic review. BMC Health Serv Res. 2018 Aug;18(1):594.

8 World Health Organization. WHO handbook for guideline development. Geneva: World Health Organization; 2014.

9 Brouwers MC, Kho ME, Browman GP, Burgers JS, Cluzeau F, Feder G, et al.; AGREE Next Steps Consortium. AGREE II: advancing guideline development, reporting and evaluation in health care. CMAJ. 2010 Dec;182(18):E839-42.

10 Shea BJ, Reeves BC, Wells G, Thuku M, Hamel C, Moran J, et al. AMSTAR 2: a critical appraisal tool for systematic reviews that include randomised or non-randomised studies of healthcare interventions, or both. BMJ. 2017 Sep;358:j4008.

11 Critical Appraisals Skills Programme. CASP checklists [Internet]. London: Critical Appraisals Skills Programme; 2021 [cited 2019 Oct 10]. Available from: https://casp-uk.net/ casp-tools-checklists/.
12 Halperin JL, Levine GN, Al-Khatib SM, Birtcher KK, Bozkurt B, Brindis RG, et al. Further evolution of the ACC/AHA Clinical Practice Guideline Recommendation Classification System: a report of the American College of Cardiology/American Heart Association Task Force on clinical practice guidelines. J Am Coll Cardiol. 2016 Apr;67(13):1572-4.

13 Niederberger M, Spranger J. Delphi technique in health sciences: a map. Front Public Health. 2020 Sep;8:457.

14 Wirth R, Dziewas R, Beck AM, Clavé P, Hamdy S, Heppner HJ, et al. Oropharyngeal dysphagia in older persons - from pathophysiology to adequate intervention: a review and summary of an international expert meeting. Clin Interv Aging. 2016 Feb;11:189-208.

15 Cohen DL, Roffe C, Beavan J, Blackett B, Fairfield CA, Hamdy S, et al. Post-stroke dysphagia: a review and design considerations for future trials. Int J Stroke. 2016 Jun;11(4):399411.

16 Powers WJ, Rabinstein AA, Ackerson T, Adeoye OM, Bambakidis NC, Becker K, et al.; American Heart Association Stroke Council. 2018 guidelines for the early management of patients with acute ischemic stroke: a guideline for healthcare professionals from the American Heart Association/American Stroke Association. Stroke. 2018 Mar;49(3): e46-110.

17 Hebert D, Lindsay MP, McIntyre A, Kirton A, Rumney PG, Bagg S, et al. Canadian stroke best practice recommendations: stroke rehabilitation practice guidelines, update 2015. Int J Stroke. 2016 Jun;11(4):459-84.

18 UK National Collaborating Centre for Chronic Conditions. Stroke: national clinical guideline for diagnosis and initial management of acute stroke and transient ischaemic attack (TIA) [Internet]. London: Royal College of Physicians; 2008 [cited 2019 Apr 29]. Available from: https://www.nice.org.uk/guidance/ng128.

19 Boulanger J, Lindsay M, Gubitz G, Smith E, Stotts G, Foley N, et al. Canadian stroke best practice recommendations for acute stroke management: prehospital, emergency department, and acute inpatient stroke care, 6th edition, update 2018. Int J Stroke. 2018;13(9):949-84.

20 Australian Stroke Foundation Clinical Guidelines for Stroke Management [Internet]. Melbourne: Stroke Foundation; 2021 [cited 2019 Aug 30]. Available from: https://informme. org.au/en/Guidelines/Clinical-Guidelinesfor-Stroke-Management.
21 Burgos R, Bretón I, Cereda E, Desport JC, Dziewas R, Genton L, et al. ESPEN guideline clinical nutrition in neurology. Clin Nutr. 2018 Feb;37(1):354-96.

22 Winstein CJ, Stein J, Arena R, Bates B, Cherney LR, Cramer SC, et al.; American Heart Association Stroke Council, Council on Cardiovascular and Stroke Nursing, Council on Clinical Cardiology, and Council on Quality of Care and Outcomes Research. Guidelines for adult stroke rehabilitation and recovery: a guideline for healthcare professionals from the American Heart Association/American Stroke Association. Stroke. 2016 Jun;47(6): e98-169.

23 Smith EE, Kent DM, Bulsara KR, Leung LY, Lichtman JH, Reeves MJ, et al.; American Heart Association Stroke Council. Effect of dysphagia screening strategies on clinical outcomes after stroke: a systematic review for the 2018 Guidelines for the early management of patients with acute ischemic stroke. Stroke. 2018 Mar;49(3):e123-8.

24 Eltringham SA, Kilner K, Gee M, Sage K, Bray $\mathrm{BD}$, Pownall S, et al. Impact of dysphagia assessment and management on risk of strokeassociated pneumonia: a systematic review. Cerebrovasc Dis. 2018;46(3-4):99-107.

25 Han TS, Lean ME, Fluck D, Affley B, Gulli G, Patel T, et al. Impact of delay in early swallow screening on pneumonia, length of stay in hospital, disability and mortality in acute stroke patients. Eur J Clin Nutr. 2018 Nov;72(11):1548-54.

26 Donovan NJ, Daniels SK, Edmiaston J, Weinhardt J, Summers D, Mitchell PH; American Heart Association Council on Cardiovascular Nursing and Stroke Council. Dysphagia screening: state of the art: invitational conference proceeding from the State-of-the-Art Nursing Symposium, International Stroke Conference 2012. Stroke. 2013 Apr;44(4):e24-31.

27 Wirth R, Smoliner C, Jäger M, Warnecke T, Leischker AH, Dziewas R; DGEM Steering Committee. Guideline clinical nutrition in patients with stroke. Exp Transl Stroke Med. 2013 Dec;5(1):14.

28 Garon BR, Sierzant T, Ormiston C. Silent aspiration: results of 2,000 video fluoroscopic evaluations. J Neurosci Nurs. 2009 Aug;41(4): 178-85.

29 Perry SE, Miles A, Fink JN, Huckabee ML. The dysphagia in stroke protocol reduces aspiration pneumonia in patients with dysphagia following acute stroke: a clinical audit. Transl Stroke Res. 2019 Feb;10(1):36-43. 
30 Hines S, Kynoch K, Munday J. Nursing Interventions for identifying and managing acute dysphagia are effective for improving patient outcomes: a systematic review update. J Neurosci Nurs. 2016 Jul-Aug;48(4):215-23.

31 Palli C, Fandler S, Doppelhofer K, Niederkorn K, Enzinger C, Vetta C, et al. Early dysphagia screening by trained nurses reduces pneumonia rate in stroke patients: a clinical intervention study. Stroke. 2017 Sep;48(9): 2583-5.

32 Middleton S, Coughlan K, Mnatzaganian G, Low Choy N, Dale S, Jammali-Blasi A, et al. Mortality reduction for fever, hyperglycemia, and swallowing nurse-initiated stroke intervention: QASC Trial (Quality in Acute Stroke Care): follow-up. Stroke. 2017 May;48(5): 1331-6.

33 Oliveira I, Couto G, Mota L. Nurses' preferred items for dysphagia screening in acute stroke patients: a qualitative study. NPT. 7(3):226233.

34 Sherman V, Flowers H, Kapral MK, Nicholson G, Silver F, Martino R. Screening for dysphagia in adult patients with stroke: assessing the accuracy of informal detection. Dysphagia. 2018 Oct;33(5):662-9.

35 Chen PC, Chuang CH, Leong CP, Guo SE, Hsin YJ. Systematic review and meta-analysis of the diagnostic accuracy of the water swallow test for screening aspiration in stroke patients. J Adv Nurs. 2016 Nov;72(11):2575-86.

36 Britton D, Roeske A, Ennis SK, Benditt JO, Quinn C, Graville D. Utility of pulse oximetry to detect aspiration: an evidence-based systematic review. Dysphagia. 2018 Jun;33(3):282-92.

37 Oliveira IJ, Mota LN, Freitas SV, Ferreira PL. Dysphagia screening tools for acute stroke patients available for nurses: a systematic review. NPT. 2019;6(3):103-15.

38 Trapl M, Enderle P, Nowotny M, Teuschl Y, Matz K, Dachenhausen A, et al. Dysphagia bedside screening for acute-stroke patients: the Gugging Swallowing Screen. Stroke. 2007 Nov;38(11):2948-52.

39 Park KD, Kim TH, Lee SH. The Gugging Swallowing Screen in dysphagia screening for patients with stroke: a systematic review. Int Nurs Stud. 2020 Jul;107:103588.

40 Boaden E, Burnell J, Hives L, Dey P, Clegg A, Lyons MW, et al. Screening for aspiration risk associated with dysphagia in acute stroke. Cochrane Database Syst Rev. 2021 Oct;10(6):CD012679.

41 Perry SE, Huckabee ML, Tompkins G, Milne $\mathrm{T}$. The association between oral bacteria, the cough reflex and pneumonia in patients with acute stroke and suspected dysphagia. J Oral Rehabil. 2020 Mar;47(3):386-94.

42 Kothari M, Pillai RS, Kothari SF, Spin-Neto R, Kumar A, Nielsen JF. Oral health status in patients with acquired brain injury: a systematic review. Oral Surg Oral Med Oral Pathol Oral Radiol. 2017 Feb;123(2):205-219.e7.
43 Dai R, Lam OL, Lo EC, Li LS, Wen Y, Mc Grath C. A systematic review and meta-analysis of clinical, microbiological, and behavioural aspects of oral health among patients with stroke. J Dent. 2015 Feb;43(2):171-80.

44 Pillai RS, Iyer K, Spin-Neto R, Kothari SF, Nielsen JF, Kothari M. Oral health and brain injury: causal or casual relation? Cerebrovasc Dis Extra. 2018;8(1):1-15.

45 Al-Khaled M, Matthis C, Binder A, Mudter J, Schattschneider J, Pulkowski U, et al.; for QugSS II Group. Dysphagia in patients with acute ischemic stroke: early dysphagia screening may reduce stroke-related pneumonia and improve stroke outcomes. Cerebrovasc Dis. 2016;42(1-2):81-9.

46 Arnold M, Liesirova K, Broeg-Morvay A Meisterernst J, Schlager M, Mono ML, et al. Dysphagia in acute stroke: incidence, burden and impact on clinical outcome. PLoS One. 2016 Feb;11(2):e0148424.

47 Schwarz M, Coccetti A, Murdoch A, Cardell E. The impact of aspiration pneumonia and nasogastric feeding on clinical outcomes in stroke patients: a retrospective cohort study. J Clin Nurs. 2018 Jan;27(1-2):e235-41.

48 Brogan E, Langdon C, Brookes K, Budgeon C, Blacker D. Respiratory infections in acute stroke: nasogastric tubes and immobility are stronger predictors than dysphagia. Dysphagia. 2014 Jun;29(3):340-5

49 Kalra L, Hodsoll J, Irshad S, Smithard D, Manawadu D; STROKE-INF Investigators. Association between nasogastric tubes, pneumonia, and clinical outcomes in acute stroke patients. Neurology. 2016 Sep;87(13):1352-9.

50 Gillman A, Winkler R, Taylor NF. Implementing the Free Water Protocol does not result in aspiration pneumonia in carefully selected patients with dysphagia: a systematic review. Dysphagia. 2017 Jun;32(3):345-61.

51 Mennella $\mathrm{H}$, Heering $\mathrm{H}$. CINAHL Nursing Guide 3: cumulative index to nursing and allied health literature. Ipswich (MA): EBSCO Publishing; 2021.

52 Baijens LW, Clavé P, Cras P, Ekberg O, Forster A, Kolb GF, et al. European Society for Swallowing Disorders - European Union Geriatric Medicine Society white paper: oropharyngeal dysphagia as a geriatric syndrome. Clin Interv Aging. 2016 Oct;11:1403-28.

53 Hua F, Xie H, Worthington HV, Furness S, Zhang Q, Li C. Oral hygiene care for critically ill patients to prevent ventilator-associated pneumonia. Cochrane Database Syst Rev. 2016 Oct;10(10):CD008367.

54 Dai R, Lam OL, Lo EC, Li LS, McGrath C. A randomized clinical trial of oral hygiene care programmes during stroke rehabilitation. J Dent. 2017 Jun;61:48-54.

55 Sørensen RT, Rasmussen RS, Overgaard K, Lerche A, Johansen AM, Lindhardt T. Dysphagia screening and intensified oral hygiene reduce pneumonia after stroke. J Neurosci Nurs. 2013 Jun;45(3):139-46.
56 Murray J, Scholten I. An oral hygiene protocol improves oral health for patients in inpatient stroke rehabilitation. Gerodontology. 2018 Mar;35(1):18-24.

57 Wagner C, Marchina S, Deveau JA, Frayne C, Sulmonte K, Kumar S. Risk of stroke-associated pneumonia and oral hygiene. Cerebrovasc Dis. 2016;41(1-2):35-9.

58 Brady M, Furlanetto D, Hunter RV, Lewis S, Milne V. Staff-led interventions for improving oral hygiene in patients following stroke. Cochrane Database Syst Rev. 2006 Oct;18(4):CD003864.

59 Ajwani S, Jayanti S, Burkolter N, Anderson C, Bhole $\mathrm{S}$, Itaoui $\mathrm{R}$, et al. Integrated oral health care for stroke patients - a scoping review. J Clin Nurs. 2017 Apr;26(7-8):891-901.

60 Kwok C, McIntyre A, Janzen S, Mays R, Teasell R. Oral care post stroke: a scoping review. J Oral Rehabil. 2015 Jan;42(1):65-74.

61 James P, Worthington HV, Parnell C, Harding $\mathrm{M}$, Lamont $\mathrm{T}$, Cheung $\mathrm{A}$, et al. Chlorhexidine mouthrinse as an adjunctive treatment for gingival health. Cochrane Database Syst Rev. 2017 Mar;3(3):CD008676.

62 Lyons M, Smith C, Boaden E, Brady MC, Brocklehurst P, Dickinson H, et al. Oral care after stroke: where are we now? Eur Stroke J. 2018 Dec;3(4):347-54.

63 Rofes L, Muriana D, Palomeras E, Vilardell N, Palomera E, Alvarez-Berdugo D, et al. Prevalence, risk factors and complications of oropharyngeal dysphagia in stroke patients: acohort study. Neurogastroenterol Motil. 2018 Mar;23(8):e13338.

64 Espuela F, Cuenca JC, Mohedas M, Sánchez JM, Cordovilla-Guardia S, Naranjo IC. Valoración nutricional y su relación con la situación funcional tras sufrir un ictus. Nutr Hosp. 2017;34(6):1353-60.

65 Gomes F, Emery PW, Weekes CE. Risk of malnutrition is an independent predictor of mortality, length of hospital stay, and hospitalization costs in stroke patients. J Stroke Cerebrovasc Dis. 2016 Apr;25(4):799-806.

66 Portugal Ministério da Saúde, Direção-Geral da Saúde. Rastreio nutricional: documento de apoio à implementação da avaliação do risco nutricional. Lisboa: Direção-Geral da Saúde; 2019.

67 Feinberg J, Nielsen EE, Korang SK, Halberg Engell K, Nielsen MS, Zhang K, et al. Nutrition support in hospitalised adults at nutritional risk. Cochrane Database Syst Rev. 2017 May;5(5):CD011598.

68 Lieber AC, Hong E, Putrino D, Nistal DA, Pan JS, Kellner CP. Nutrition, energy expenditure, dysphagia, and self-efficacy in stroke rehabilitation: a review of the literature. Brain Sci. 2018 Dec;8(12):e218.

69 Ojo O, Brooke J. The use of enteral nutrition in the management of stroke. Nutrients. 2016 Dec;8(12):e827.

70 Nascimento A, Carvalho M, Nogueira J, Abreu P, Nzwalo H. Complications associated with nasogastric tube placement in the acute phase of stroke: a systematic review. J Neurosci Nurs. 2018 Aug;50(4):193-8. 
71 Rowat A. Enteral tube feeding for dysphagic stroke patients. Br J Nurs. 2015 Feb;24(3):138 45.

72 López-Gómez JJ, Delgado-García E, CotoGarcía C, Torres-Torres B, Gómez-Hoyos E, Serrano-Valles C, et al. Influence of hyperglycemia associated with enteral nutrition on mortality in patients with stroke. Nutrients. 2019 Apr;11(5):e996.

73 Ojo O, Keaveney E, Wang XH, Feng P. The effect of enteral tube feeding on patients' health-related quality of life: a systematic review. Nutrients. 2019 May;11(5):1046.

74 Eltringham SA, Kilner K, Gee M, Sage K, Bray BD, Smith CJ, et al. Factors associated with risk of stroke-associated pneumonia in patients with dysphagia: a systematic review. Dysphagia. 2020 Oct;35(5):735-44.

75 Kim G, Baek S, Park HW, Kang EK, Lee G. Effect of nasogastric tube on aspiration risk: results from 147 patients with dysphagia and literature review. Dysphagia. 2018 Dec;33(6) 731-8.

76 Kwak HJ, Kim L, Ryu BJ, Kim YH, Park SW, Cho DG, et al. Influence of nasogastric tubes on swallowing in stroke patients: measuring hyoid bone movement with ultrasonography. Ann Rehabil Med. 2018 Aug;42(4):551-9.

77 Dennis M, Lewis S, Cranswick G, Forbes J; FOOD Trial Collaboration. FOOD: a multicentre randomised trial evaluating feeding policies in patients admitted to hospital with a recent stroke. Health Technol Assess. 2006;10(2):iii-iv, ix-x, 1-120.

78 Geeganage C, Beavan J, Ellender S, Bath PM. Interventions for dysphagia and nutritional support in acute and subacute stroke. Cochrane Database Syst Rev. 2012 Oct;10: CD000323.

79 Bath PM, Lee HS, Everton LF. Swallowing therapy for dysphagia in acute and subacute stroke. Cochrane Database Syst Rev. 2018 Oct;10(10):CD000323.

80 Chiang CF, Lin MT, Hsiao MY, Yeh YC, Liang YC, Wang TG. Comparative efficacy of noninvasive neurostimulation therapies for acute and subacute poststroke dysphagia: a systematic review and network meta-analysis. Arch Phys Med Rehabil.2019Apr;100(4):739_ 750.e4.

81 Tian ZY, Liao X, Gao Y, Liang SB, Zhang CY, $\mathrm{Xu} \mathrm{DH}$, et al. An overview of systematic reviews and meta-analyses on acupuncture for post-acute stroke dysphagia. Geriatrics (Basel). 2019 Dec;4(4):e68

82 Chen YW, Chang KH, Chen HC, Liang WM, Wang YH, Lin YN. The effects of surface neuromuscular electrical stimulation on poststroke dysphagia: a systemic review and metaanalysis. Clin Rehabil. 2016 Jan;30(1):24-35.
83 Brady S, Donzelli J. The modified barium swallow and the functional endoscopic evaluation of swallowing. Otolaryngol Clin North Am. 2013 Dec;46(6):1009-22.

84 Coleman ER, Moudgal R, Lang K, Hyacinth HI, Awosika OO, Kissela BM, et al. Early rehabilitation after stroke: a narrative review. Curr Atheroscler Rep. 2017 Nov;19(12):59.

85 Stinear C, Ackerley S, Byblow W. Rehabilitation is initiated early after stroke, but most motor rehabilitation trials are not: a systematic review. Stroke. 2013 Jul;44(7):2039-45.

86 Bakhtiyari J, Sarraf P, Nakhostin-Ansari N, Tafakhori A, Logemann J, Faghihzadeh S, et al. Effects of early intervention of swallowing therapy on recovery from dysphagia following stroke. Iran J Neurol. 2015 Jul;14(3):11924.

87 Kim HH, Park JS. Efficacy of modified chin tuck against resistance exercise using handfree device for dysphagia in stroke survivors: a randomised controlled trial. J Oral Rehabil. 2019 Nov;46(11):1042-6.

88 Simonelli M, Ruoppolo G, Iosa M, Morone G, Fusco A, Grasso MG, et al. A stimulus for eating. The use of neuromuscular transcutaneous electrical stimulation in patients affected by severe dysphagia after subacute stroke: a pilot randomized controlled trial. NeuroRehabilitation. 2019;44(1):103-10

89 Tarameshlu M, Ansari NN, Ghelichi L, Jalaei S. The effect of repetitive transcranial magnetic stimulation combined with traditional dysphagia therapy on poststroke dysphagia: a pilot double-blinded randomized-controlled trial. Int J Rehabil Res. 2019 Jun;42(2):133-8.

90 Wu W-B, Fan D-F, Zheng C, Que B-F, Lian Q-Q, Qiu R, et al. Relieving throat and opening orifice acupuncture therapy for the poststroke dysphagia. World J Acupunct Moxibustion. 2019;29(1):37-41.

91 Garcia JM, Chambers E 4th. Managing dysphagia through diet modifications. Am J Nurs. 2010 Nov;110(11):26-33.

92 McCurtin A, Boland P, Kavanagh M, Lisiecka D, Roche C, Galvin R. Do stroke clinical practice guideline recommendations for the intervention of thickened liquids for aspiration support evidence based decision making? A systematic review and narrative synthesis. J Eval Clin Pract. 2020 Dec;26(6):1744-60.

93 Beck AM, Kjaersgaard A, Hansen T, Poulsen I. Systematic review and evidence based recommendations on texture modified foods and thickened liquids for adults (above 17 years) with oropharyngeal dysphagia - an updated clinical guideline. Clin Nutr. 2018 Dec;37(6 6 Pt A):1980-91.
94 Newman R, Vilardell N, Clavé P, Speyer R. Effect of bolus viscosity on the safety and efficacy of swallowing and the kinematics of the swallow response in patients with oropharyngeal dysphagia: white paper by the European Society for Swallowing Disorders (ESSD). Dysphagia. 2016 Apr;31(2):232-49.

95 Crary MA, Carnaby GD, Shabbir Y, Miller L, Silliman S. Clinical variables associated with hydration status in acute ischemic stroke patients with dysphagia. Dysphagia. 2016 Feb;31(1):60-5.

96 Miles A, McFarlane M, Scott S, Hunting A. Cough response to aspiration in thin and thick fluids during FEES in hospitalized inpatients. Int J Lang Commun Disord. 2018 Sep;53(5):909-18.

97 Wright DJ, Smithard DG, Griffith R. Optimising medicines administration for patients with dysphagia in hospital: medical or nursing responsibility. Geriatrics (Basel). $2020 \mathrm{Feb} ; 5(1): \mathrm{e} 9$.

98 McCurtin A, Healy C, Kelly L, Murphy F, Ryan J, Walsh J. Plugging the patient evidence gap: what patients with swallowing disorders post-stroke say about thickened liquids. Int J Lang Commun Disord. 2018 Jan;53(1):30-9.

99 Barker A, Doeltgen S, Lynch E, Murray J. Perceived barriers and enablers for implementing water protocols in acute stroke care: a qualitative study using the Theoretical Domains Framework. Int J Speech Lang Pathol. 2019 Jun;21(3):286-94.

100 Steele CM, Alsanei WA, Ayanikalath S, Barbon CE, Chen J, Cichero JA, Coutts K, Dantas RO, Duivestein J, Giosa L, Hanson B, Lam P, Lecko C, Leigh C, Nagy A, Namasivayam AM, Nascimento WV, Odendaal I, Smith $\mathrm{CH}$, Wang $\mathrm{H}$. The influence of food texture and liquid consistency modification on swallowing physiology and function: a systematic review. Dysphagia. 2015 Feb;30(1):2-26.

101 Swan K, Speyer R, Heijnen BJ, Wagg B, Cordier R. Living with oropharyngeal dysphagia: effects of bolus modification on healthrelated quality of life - a systematic review. Qual Life Res. 2015 Oct;24(10):2447-56.

102 Bolivar-Prados M, Rofes L, Arreola V, Guida S, Nascimento WV, Martin A, Vilardell N, Ortega Fernández O, Ripken D, Lansink M, Clavé P. Effect of a gum-based thickener on the safety of swallowing in patients with poststroke oropharyngeal dysphagia. Neurogastroenterol Motil. 2019 Nov;31(11): e13695.103.

103 Vilardell N, Rofes L, Arreola V, Speyer R, Clavé P. A comparative study between modified starch and xanthan gum thickeners in post-stroke oropharyngeal dysphagia. Dysphagia. 2016 Apr;31(2):169-79. 